\title{
Does Space Have a Gravitational Susceptibility? A Model for the $\Lambda$ CDM Density Parameters in the Friedmann Equation
}

\author{
Christopher Pilot \\ Physics, Maine Maritime Academy, Castine, ME, USA (Retired) \\ Email: chris.pilot43@gmail.com
}

How to cite this paper: Pilot, C. (2021) Does Space Have a Gravitational Susceptibility? A Model for the $\Lambda$ CDM Density Parameters in the Friedmann Equation. Journal of High Energy Physics, Gravitation and Cosmology, 7, 578-507. https://doi.org/10.4236/jhepgc.2021.72028

Received: December 24, 2020

Accepted: April 5, 2021

Published: April 8, 2021

Copyright $\odot 2021$ by author(s) and Scientific Research Publishing Inc. This work is licensed under the Creative Commons Attribution International License (CC BY 4.0).

http://creativecommons.org/licenses/by/4.0/

\begin{abstract}
We propose a model for gravity based on the gravitational polarization of space. With this model, we can relate the density parameters within the Friedmann model, and show that dark matter is bound mass formed from massive dipoles set up within the vacuum surrounding ordinary matter. Aggregate matter induces a gravitational field within the surrounding space, which reinforces the original field. Dark energy, on the other hand, is the energy density associated with gravitational fields both for ordinary matter, and bound, or induced dipole matter. At high CBR temperatures, the cosmic susceptibility, induced by ordinary matter vanishes, as it is a smeared or average value for the cosmos as a whole. Even though gravitational dipoles do exist, no large-scale alignment or ordering is possible. Our model assumes that space, i.e., the vacuum, is filled with a vast assembly (sea) of positive and negative mass particles having Planck mass, called planckions, which is based on extensive work by Winterberg. These original particles form a very stiff two-component superfluid, where positive and negative mass species neutralize one another already at the submicroscopic level, leading to zero net mass, zero net gravitational pressure, and zero net entropy, for the undisturbed medium. It is theorized that the gravitational dipoles form from such material positive and negative particles, and moreover, this causes an intrinsic polarization of the vacuum for the universe as a whole. We calculate that in the present epoch, the smeared or average susceptibility of the cosmos equals, $\overline{\chi_{0}}=0.842$, and the overall resulting polarization equals, $\overline{P_{0}}=2.396 \mathrm{~kg} / \mathrm{m}^{2}$. Moreover, due to all the ordinary mass in the universe, made up of quarks and leptons, we calculate a net gravitational field having magnitude, $\overline{g^{(0)}}=3.771 \mathrm{E}-10 \mathrm{~m} / \mathrm{s}^{2}$. This smeared or average value permeates all of space, and can be deduced by any observer, irrespective of location within the
\end{abstract}


universe. This net gravitational field is forced upon us by Gauss's law, and although technically a surface gravitational field, it is argued that this surface, smeared value holds point for point in the observable universe. A complete theory of gravitational polarization is presented. In contrast to electrostatics, gravistatics leads to anti-screening of the original source field, increasing the original value, $\overrightarrow{g^{(0)}}$, to, $\vec{g}=\overrightarrow{g^{(0)}}+\overrightarrow{g^{(1)}}>\overrightarrow{g^{(0)}}$, where $\overrightarrow{g^{(1)}}$ is the induced or polarized field. In the present epoch, this leads to a bound mass, $M_{B}=\overline{\chi_{0}} / \overline{K_{0}} M_{F}=5.33 M_{F}$, where $M_{F}$ is the sum of all ordinary source matter in the universe, and $\overline{K_{0}}$ equals the relative permittivity. A new radius, and new mass, for the observable universe is dictated by the density parameters in Friedmann's equation, and Gauss's law. These lead to the very precise values, $R_{0}=3.217 \mathrm{E} 27$ meters, and, $M_{F}=5.847 \mathrm{E} 55 \mathrm{~kg}$, respectively, somewhat larger than current less accurate estimates.

\section{Keywords}

Extended Gravitational Model, Friedmann Equation, Dark Matter,

Dark Energy, Vacuum Energy, Winterberg Model,

Gravitational Polarization Model, Planck Mass Model

\section{Introduction}

Dark matter and dark energy are phenomena which at present are poorly understood. In particular, no suitable model exists for predicting the underlying density parameters, $\left(\Omega_{b}, \Omega_{c}, \Omega_{\Lambda}\right)$, associated with their relative energy content. We have the numerical values in the current epoch, $\left(\Omega_{b}, \Omega_{c}, \Omega_{\Lambda}\right)=(0.0486,0.2589,0.6911)$, obtained from the latest $\Lambda \mathrm{CDM}$ data release [1] [2] [3]. We also have a sense for how these quantities scale as the universe expands. A formal theory, however, connecting the quantities above has yet to be formulated. Also, are dark matter and dark energy related? They seem totally different in character and thus, it is difficult to imagine that a more intimate connection between the two should even exist. Nonetheless, we believe such a connection does, in fact, exist, which will be demonstrated in this paper. In short, we present a theory relating all three ideas, dark matter, dark energy, and their interconnection.

Some time ago, it has been astutely observed by Hajdukovic, in a series of papers [4] [5] [6] [7], that the polarization of space may provide, in some sense, the key towards an understanding of dark matter. He later added dark energy, as well. Two facts stand out. The first is that gravitational polarization may provide an anti-screening feature giving a total mass, which is the sum of free source mass and bound mass, a larger value. This could help account for dark matter, and the many observations associated with such. Moreover, virtual particle creation/annihilation may provide the background vacuum energy needed to explain 
dark energy. He was thinking specifically, of virtual pion pair production/annihilation within the vacuum where the masses equal, $m\left(\pi^{ \pm}\right)=2.488 \mathrm{E}-28 \mathrm{~kg}$. The gravitational force, being attractive, could form a polarization cloud about ordinary matter leading to larger than expected mass. This could explain rotation curves, the halo effect of galaxies, the motion of galaxies within superclusters of galaxies, gravitational lensing, etc. Virtual particle, $\pi^{ \pm}$creation/annihilation, on the other hand, could fill the rest of space creating an aether-like medium for dark energy, and rendering an energy density compatible with dark energy. The theory, however, never gained significant traction, as specific predictions were scarce, and some details were sketchy.

Another intriguing notion relating to vacuum energy and dark energy was presented by Winterberg, who, in a series of papers [8]-[13], and in a book [14], forwarded the novel idea that space is filled with a vast assembly (sea) of positive and negative mass particles, which he called planckions. They have Ur-mass, or original mass, of positive and negative the Planck mass where,

$\pm M_{P l}= \pm 2.176 \mathrm{E}-8 \mathrm{~kg}$. These particles were formed presumably at the Planck temperature, $T_{P l} \sim 10^{32}$ Kelvin. He developed a very detailed model regarding the interaction of such Planckion particles where like masses repel each other, and unlike masses are forced close to one other. The two species of submicroscopic masses form a very stiff two-component superfluid, which, as a whole, is massively neutral due to their mass compensating effect. Each positive and negative mass keeps the other masses, within their species, a fixed distance apart within this two-component superfluid. Also, the speed of sound (phonons) within such a dense aether-like medium was shown to be equal to the speed of light.

In his theory, Winterberg offers an explanation for the tremendous vacuum energy in the earliest epochs [15]. He derives quantum mechanics, and the general theory of relativity, as two asymptotic limits within his more general model. What is interesting is that his theory is totally mechanistic/atomistic in its approach, along the lines of Boltzmann, when he developed his statistical mechanics. Space or vacuum is not empty, but rather consists of a medium made up of these positive and negative mass particles. Vortices and other stable disturbances/distortions within this two-component superfluid produce quasi-particles, which he identifies with elementary particles, and their mediating fields. Needless to say, his theory is a very ambitious one, developed as an alternative to string theory.

Winterberg's theory is very detailed in its calculations, and somewhat complex, in this author's opinion, especially as it relates to the formation of quasi-particles (collective excitations) within the vacuum. He does not adequately address what happens to his two-component superfluid upon expansion of the universe, and his explanations for dark matter and dark energy are superficial. He focuses primarily on the vacuum energy, the fluid forces acting on the planckions, the formation of quasi-particles within the superfluid, the underlying symmetry, which is, $S U(2) \sim S O(3)$, versus Lorentz invariance, as the fundamental symmetry of nature, and a derivation of quantum mechanics and the general theory of relativity as special asymptotic limits. 
In spite of its complexity, however, we feel that his theory has many inherent merits. The mass compensating effect, already at the sub-microscopic level $\left(<10^{-18}\right.$ meters) leads to zero net mass, zero net gravitational pressure, and zero net entropy for undisturbed space, which is seemingly empty. Elastic collisions with CBR blackbody photons will cause inherent fluctuations and oscillations of the positive and negative masses, leading to an uncertainty in position and momentum for any material particle placed upon, or within, such a space, much as a ship rocks to and fro when placed upon the ocean. The Schrödinger equation is also derived from first principles using Boltzmann-type arguments. The inter-planckion forces lead to a very stiff medium causing disturbances (phonons) to move at the speed of light. The whole notion of the vacuum as a condensed medium, where the laws from condensed matter physics can be brought to bear is worth mentioning. In contrast to Hajdukovic's theory, his particles are very real, and not virtual, $\pi^{ \pm}$pairs. He also introduces, in contrast to Hajdukovic, like massive particles which repel, and opposite mass particles, which attract, albeit indirectly.

We wish to build upon Winterberg's model further in this paper. The model we present here, and develop further, is really a combination of the both the Winterberg model, and that of Hajdukovic. We borrow heavily from each, in order to develop a new and independent theory, which may help explain the cosmological density parameters associated with dark matter and dark energy. It is our belief that dark matter and dark energy are related to one another on a fundamental level. One major modification that we will introduce, which is different from Winterberg, is that the vacuum is more of a supersolid, versus a superfluid. Supersolids are now known to exist [16]-[22], but only at extreme low temperatures. We imagine something similar happening, but at much higher temperatures, and for a different type of mass. Instead of atoms, we are looking at planckion mass, and planckion mass condensates. Also, there is no limit to the temperature of a super solid; it can be very high if the pressures, densities, and/or interactions are correspondingly high.

This is actually a first in a series of papers where we intend to look at the macroscopic, and microscopic, implications of such a theory. This includes deriving the scaling laws for both macroscopic and microscopic variables upon expansion of the universe. This work will start the process. Here, we focus exclusively on the present epoch, and explain the numerical values associated with the density parameters. We also predict new, and very precise, values for the Hubble radius, and mass of the observable universe, within this radius. Extended gravity theories are not new, and many theories have surfaced which propose an explanation for dark matter and dark energy [23]. Ours is but one example.

The outline of this paper is as follows. In Section II, we review the fluid forces acting on the individual planckions, and prove that the positive and negative mass planckions do not interact directly. Both arguments are due to Winterberg. We then take this a step further, and introduce specific restoring forces acting on 
individual planckions. For the polarization of space, the planckions need to be spatially anchored, or locked in position. If displaced from their equilibrium position, specific restoring forces must apply. External influences are external gravitational fields produced by source matter, and temperature driven CBR photon collisions. In Section III, we develop a theory of gravistatics, much along the lines of electrostatics. There are some notable differences such as anti-screening in gravistatics, versus screening in electro-statics, which we make clear. We define key relations, such as bound mass, and susceptibility, which are needed for the following sections.

In Section IV, our identifications are made for dark matter and dark energy. We will relate both concepts formally through their underlying equations, and predict a new radius, and a new mass, for the observable Hubble bubble. These new values are forced upon us by the current values of the $\Lambda$ CDM density parameters, and Gauss's law. Gauss's law is treated in detail in Section III. It is our contention that the universe has a net smeared gravitational field, and a net smeared polarization due to all the free mass contained within it. The former is a given, whereas the latter is an assumption. We calculate the various gravitational field values, as well as other quantities relating to the cosmos as a whole, when distance scales greater than $100 \mathrm{Mpc}$ are considered. In Section V, we present our summary and conclusions.

\section{The Winterberg Model and Extension}

In this section we focus on the anchoring of planckions in space. As a prerequisite for defining susceptibility and polarization, the material Planck particles, i.e., planckions need to be locked in position, spatially, in some sense.

We start with the fluid forces governing the positive and negative mass planckion particles due to Winterberg. In the current cosmological epoch, we assume that their masses equal positive and negative the Planck mass, $\pm M_{P l}$, where $\pm M_{P l}= \pm 2.176 \mathrm{E}-8 \mathrm{~kg}$. All units not explicitly written out are MKS units. Following Winterberg, we assume that like masses within the fluid, whether they possess positive or negative mass, will repel each other. Unlike mass planckions will attract one another, but indirectly.

The fluid force acting on a positive mass planckion is due to the other positive mass planckions comprising the fluid. It is

$$
\overrightarrow{F_{+}}=-n_{+}^{-1} \vec{\nabla} p_{+}=-n_{+}^{-1}\left(\vec{\nabla} n_{+}\right) M_{P l} c^{2}
$$

In Equation (2-1), $n_{+}(\vec{x})$ stands for the positive mass planckion number density, and $\vec{\nabla} p_{+}$is the gradient of the gravitational pressure, $p_{+}(\vec{x})$. The positive gravitational pressure exerted by the other positive mass planckions is defined as, $p_{+} \equiv n_{+} M_{P l} c^{2}$, where $c$ is the speed of light and, $M_{P l}$, is the Planck mass.

For the negative Planck mass particle, there is a similar force. Here, the fluid force acting on the negative mass planckion equals, 


$$
\overrightarrow{F_{-}}=+n_{-}^{-1} \vec{\nabla} p_{-}=+n_{-}^{-1}\left(\vec{\nabla} n_{-}\right)\left(-M_{P l}\right) c^{2}
$$

This force, $\overrightarrow{F_{-}}$, is due to the other negative mass planckions populating the vacuum. We note that we have positive the gradient in this expression. A negative mass particle will want to take the path of steepest ascent in a pressure field. Think of a negative mass in the earth's gravitational field. When released, it would accelerate upwards towards higher gravitational pressure. Here, $n_{-}(\vec{x})$ stands for the negative mass planckion number density, and, $p_{-}(\vec{x})$ is the corresponding negative mass gravitational pressure, defined by,

$p_{-} \equiv n_{-}\left(-M_{P l}\right) c^{2}=-n_{-} M_{P l} c^{2}$. We notice that $p_{-}$is inherently negative.

For the undisturbed fluid (vacuum) with no gravitational fields, the positive and negative mass number densities balance, i.e., $n_{+}(\vec{x})=n_{-}(\vec{x})$. The total gravitational pressure becomes,

$$
p=p_{+}+p_{-}=M_{P l} c^{2}\left(n_{+}-n_{-}\right)=0 \quad \text { (undisturbed fluid) }
$$

The total planckion mass density equals,

$$
\rho=\rho+\rho_{-}=M_{P l}\left(n_{+}-n_{-}\right)=0 \quad \text { (undisturbed fluid) }
$$

We notice that, by definition, the component mass densities are both positive and negative,

$$
\rho_{+} \equiv M_{P l} n_{+}(\vec{x}), \quad \rho_{-} \equiv-M_{P l} n_{-}(\vec{x})
$$

Moreover, upon comparing Equation (2-4), with Equation (2-3), it is clear that,

$$
p_{+}=\rho_{+} c^{2}, \quad p_{-}=\rho_{-} c^{2}, \quad p=\rho c^{2}
$$

The equations of state for planckions are straightforward, as, $p=w \rho c^{2}$, with $w=1$. This holds for the component parts as well, $p_{+}=w \rho_{+} c^{2}$, and, $p_{-}=w \rho_{-} c^{2}$, where, $w=1$. Number density equilibrium in a region of space, $n_{+}(\vec{x})=n_{-}(\vec{x})$, will also be maintained if planckions are oscillating or vibrating due to collisions with CBR blackbody photons. If, however, there is an applied gravitational field, $n_{+}(\vec{x})$ will be greater than, $n_{-}(\vec{x})$. Then we have a net gravitational pressure, and a net mass density, which is now unequal to zero. A net planckion mass density is a new prediction of Winterberg's theory.

The positive and negative mass planckions are anchored in position, spatially, due to their mutual repulsion and attraction. If brought too close together, planckions of the same species will repel, and if they are separated by too large a distance, they will attract. In this way equilibrium is maintained, where a fixed distance of separation is maintained between particles of the same species. To see this more clearly, we look at a one-dimensional version of Equation (2-1). This reads

$$
F_{+, x}=-n_{+}^{-1} \mathrm{~d} n_{+} / \mathrm{d} x M_{P l} c^{2}
$$

The negative sign indicates a restoring force, because as the gravitational pressure, $p_{+}=n_{+} M_{P l} c^{2}$, increases, in going from $x$, to $x+\mathrm{d} x$, this will produce a force acting in the opposite direction tending to bring the particle back to equilibrium. Hence, we replace the left hand side by, $F_{x}=-\kappa x$, where, $\kappa$ is 
the spring constant, and, $x=0$, is the equilibrium position. Substituting this into Equation (2-7), we now obtain,

$$
-\kappa x=-n_{+}^{-1} \mathrm{~d} n_{+} / \mathrm{d} x M_{P l} c^{2}
$$

Bringing the $\mathrm{d} x$ over to the left hand side, and integrating both sides, from, $x=0$, to, $x$, gives

$$
n_{+}(x)=n_{+}(0) \mathrm{e}^{\kappa x^{2} /\left(2 M_{P I} c^{2}\right)}
$$

Increasing $x$ in either the positive or negative sense, increases the number density, $n_{+}$, but also at the same time, the mass density, $\rho_{+}$, and the gravitational pressure, $p_{+}$. See Equations (2-5), and (2-6). This produces a force acting in the opposite direction, as indicated by Equation (2-7). Equation (2-9), indicates a "hole", or trough, centered about, $x=0$.

For negative mass planckions, for a bounded, i.e., sinusoidal solution, the corresponding restoring force law must obey,

$$
\left(-M_{P l}\right) \ddot{x}=+\kappa x
$$

Here, the value of the spring constant, $\kappa$, is assumed to be the same as for the positive mass planckion particle. And so, $F_{x}=+\kappa x$, for the negative mass particle. Otherwise we would have a hyperbolic-sinusoidal solution which is unbounded for the negative mass planckion. We substitute Equation (2-10), on the left hand side of Equation (2-2), after specializing to the one-dimensional case. Proceeding as above, we find that,

$$
n_{-}(x)=n_{-}(0) \mathrm{e}^{-\kappa x^{2} /\left(2 M_{P I} c^{2}\right)}
$$

This Gaussian looking function indicates a peak at, $x=0$, versus a trough, as in Equation (2-9). A peak for a negative mass particle is equivalent to a "hole", for a positive mass particle. In other words, a negative mass planckion will move such as to increase its gravitational pressure. Think of a negative mass particle in the earth's gravitational field. When released it would accelerate upwards at, 9.81 $\mathrm{m} / \mathrm{s}^{2}$, increasing its gravitational pressure. At, $x=0$, in Equation (2-11), we have maximum pressure for a negative mass planckion. Any positive or negative displacement from this equilibrium position, will lead to restoring forces tending to bring the negative mass particle back to, $x=0$. Equations (2-9), and (2-11), are new equations.

What about positive mass and negative mass planckions? They will repel each other if spaced too close together, and attract each other if separated by too large a distance. They will do so, indirectly. This can be seen as follows [14].

Assume an elastic collision between a positive and negative mass planckion. Conservation of energy demands that,

$$
v_{1}^{2}-v_{2}^{2}=v_{1}^{\prime 2}-v_{2}^{\prime 2}
$$

The $1 / 2 M_{P l}$ factors out between the left and the right hand sides. Due to their hefty mass, a non-relativistic treatment is warranted. The unprimed (primed) velocities stand for the velocities before (after) the collision. Conservation of 
momentum, on the other hand, requires that,

$$
\overrightarrow{v_{1}}-\overrightarrow{v_{2}}=\overrightarrow{v_{1}^{\prime}}-\overrightarrow{v_{2}^{\prime}}
$$

Divide the left hand side of Equation (2-12), by the left hand side of Equation (2-13), and do the same on the right hand side. This gives

$$
\overrightarrow{v_{1}}+\overrightarrow{v_{2}}=\overrightarrow{v_{1}^{\prime}}+\overrightarrow{v_{2}^{\prime}}
$$

Next, we add the left hand side of Equation (2-13), to the left hand side of Equation (2-14). We do the same on the right hand side, and we obtain

$$
2 \overrightarrow{v_{1}^{\prime}}=2 \overrightarrow{v_{1}}
$$

Subtracting the left hand side of Equation (2-13), from the left hand side of Equation (2-14), and doing the same on the right hand side renders

$$
2 \overrightarrow{v_{2}^{\prime}}=2 \overrightarrow{v_{2}}
$$

Equations (2-15), and, (2-16), tell us that the two planckions of opposite mass go through each other as if the other mass weren't there. This proves that the positive and negative mass planckions do not interact directly.

They do, however, interact indirectly. Seeing that the positive and negative species occupy the same space, and that each particle within their respective species keeps a fixed distance from other particles of the same species, the positive and negative planckions are invariably drawn next to each other. In other words, they seemingly attract one another, but this is more a consequence of fluid forces acting on each separate species.

In Equations (2-9), and, (2-11), we have buried within the exponent, an inherent frequency,

$$
\omega^{2}=\kappa / M_{P l}
$$

In this equation, $\omega$, is the oscillating frequency of planckions when displaced. We will see in a later work that bombardment with CBR photons can set the positive and negative mass planckions vibrating, or oscillating. The frequency of the bombarding photons will turn out to equal the frequency of the oscillating positive and negative mass planckions. Since we have a spectrum of frequencies at a particular CBR temperature, we will also have a spectrum of oscillating frequencies for the positive and negative mass particles, which make up the vacuum.

We also believe that $M_{P l}$ may not be a true constant of nature, which would mean that the Newton's gravitational constant, $G$, varies with cosmological time [24] [25] [26]. Hence, if $G$ scales, then, $M_{P l} \equiv(\hbar c / G)^{1 / 2}$, would also scale as the universe expands. In this work, we concern ourselves with the present epoch only, and therefore, $G$ has the fixed value, $G=6.674 \mathrm{E}-11(\mathrm{MKS})$. This gives, $M_{P l}=2.176 \mathrm{E}-8 \mathrm{~kg}$, using the equation above. Irrespective of whether $M_{P l}$ scales or not, Equation (2-17), will hold.

In Equation (2-17), we can pick out a particular frequency, for example, the most probable frequency. According to Planck's blackbody radiation formula, 
that peak frequency is directly proportional to temperature, and is given by the following expression,

$$
v_{\text {peak }}=\alpha k_{B} T / h=(2.8214 \cdots) k_{b} T / h=(5.879 \mathrm{E} 10) T
$$

In Equation (2-18), $\alpha=2.8214 \cdots$, is a numerical constant, $h$ is Planck's constant, and $k_{B}$ equals Boltzmann's constant. The angular frequency is, of course, $\omega=2 \pi v$. We choose the temperature to be the CBR temperature. Using these facts, the $\omega^{2}$ must scale as CBR temperature squared. In other words,

$$
\omega^{2} / \omega_{0}^{2}=T^{2} / T_{0}^{2}=\left(R_{0} / R\right)^{2}=(1+z)^{+2}=a^{-2}
$$

In this equation, $z$ is the redshift, $T$ the CBR temperature, and " $a$ " is the cosmic scale parameter. We are using the convention, where in the current era, $a_{0}=1$, and the subscript " 0 " on variables denotes the present epoch. Variables without a subscript refer to a different cosmological era. The radius, $R$, stands for the Hubble radius, the radius of the observable universe.

Upon comparison with Equation (2-17), we can therefore claim that Equation (2-19), can be re-expressed as,

$$
\left(\kappa / M_{P l}\right)\left(M_{P l, 0} / \kappa_{0}\right)=a^{-2}
$$

Or, what is equivalent,

$$
\left(\kappa / \kappa_{0}\right)=\left(M_{P l} / M_{P l, 0}\right) a^{-2}=\left(G_{0} / G\right)^{1 / 2} a^{-2}
$$

If Newton's constant is a true constant of nature, then obviously, $G=G_{0}$, and we are left with a much simpler scaling law for $\kappa$. The point is that, as one goes back in cosmological time, the planckion spring constant, $\kappa$, actually increases. The fluid was stiffer in earlier epochs, a somewhat surprising result! If $G$ does scale, then we have an added contribution due to, $\left(G_{0} / G\right)^{1 / 2}$, but this is not nearly as strong as $a^{-2}$, which is the dominant term. This means that, for a particular displacement, the gravitational pressure restoring the planckion back to its equilibrium position will be larger in prior epochs. Even though this is the case, $\kappa$, in the present era is still incredibly large, leading to a very stiff aether. The $\kappa$ value will actually be calculated in a follow-up paper.

A full blown treatment of harmonic oscillations for the planckions will be given in subsequent work. Incidentally, the center of mass for an oscillating positive and negative Planck dipole pair is at spatial infinity. In other words, both the positive and negative masses will oscillate about a point at, $\infty$.

Anchoring of particles comprising the vacuum is important for many reasons. First, we have a quasi-crystallization of space. A lattice type structure for space emerges, where the individual particles which make up that space are, more or less, locked in position. Hence, we are thinking in terms of a super-solid, or an extremely stiff superfluid. Also, through the general theory of relativity, an energy-momentum stress tensor will lead literally to a mechanical deformation of this space. Second, any polarization of space has, as a prerequisite, that the individual particles are ordered and configured in some sense. This is only possible when the particles are spatially fixed in position. Third, anchoring is important 
for the speed of light. We require a very stiff medium, or aether, for the propagation of light. As shown by Winterberg, the speed of sound (phonons) within this two-component superfluid equals the speed of light. The vacuum, in our view, is a super-solid/superfluid, made up of planckion particles, which form a rigid, or semi-rigid, spatially ordered structure. Even though the planckions are anchored in position, producing a lattice super-solid, there is no viscosity.

We will consider two external influences, which will cause deviations about the equilibrium position. The first is due to CBR blackbody photon bombardment. This is temperature driven and will lead to harmonic oscillations about the minimum gravitational potential well position, $x=0$. Technically, the oscillation is about, $x=\infty$, if we consider an oscillating positive and negative mass dipole pair, but the effect is the same. For the negative mass particles, the oscillations are about the peak gravitational pressure positions, $x=0$. Blackbody photons can and will interact with the planckions through collisions, i.e., impart energy and momentum to our individual planckions causing them to oscillate about, $x=0$, or $x=\infty$ when considering a dipole pair.

This bombardment by the blackbody photons produces a Zitterbewegung, an erratic, chaotic motion, because the vacuum itself is now oscillating/vibrating. Any material particle placed within such a space will have an inherent rocking to and fro motion, much like a ship when placed on the ocean. This produces a situation where position and momentum cannot be determined simultaneously. In other words, the Heisenberg indeterminacy principle has its explanation, or interpretation, in underlying planckion motion, as first proposed by Winterberg. The CBR temperature thus produces a vacuum made up of a vast assembly (sea) of harmonically oscillating, and vibrating planckions, all with differing frequencies.

Temperature induced massive dipoles will form, but this can lead to no net macroscopic polarization of space, even at low CBR temperatures, due to the random and chaotic nature of blackbody radiation. The collisions between blackbody photons and planckions will be random, chaotic and erratic. Even though a non-vanishing dipole, $p_{\delta}=M_{P l}(2 \delta)$, exists for the oscillating positive and negative mass pair, where $\delta$ is the rms (root mean square) amplitude, no single direction in space can be singled out. Thus, $\left\langle\overrightarrow{p_{\delta}}\right\rangle=0$, or, $\sum \overrightarrow{p_{\delta, j}}=0$, in any macroscopic region of space. The factor of two, above, is due to simultaneous displacement of positive and negative mass.

A second external influence is the gravitational field. A localized source mass distribution in space, $\rho_{F}(\vec{x})$, will produce a gravitational field, $\overrightarrow{g^{(0)}}(\vec{x})$, which surrounds it and possesses the same symmetry as, $\rho_{F}(\vec{x})$. The mass density, $\rho_{F}(\vec{x})$, refers to free, or ordinary source matter, i.e., matter made up of atoms and molecules, such as, gases, dust, aggregate solids and liquids, stars, galaxies, etc. The gravitational field, $\overrightarrow{g^{(0)}}(\vec{x})$, will lead to polarization of the surrounding medium in our model, if conditions are right (strong enough applied field, not too extreme temperature, enough of a dipole moment, etc.). Then the vacuum becomes polarized, where the polarization equals, $\vec{P}(\vec{x})=\varepsilon \chi \vec{g}(\vec{x})$, an expres- 
sion familiar from electrostatics, but now applied to gravistatics. The, $\varepsilon$, in the polarization equation, is the gravitational permittivity of the vacuum, and $\chi$ is the gravitational susceptibility.

In gravity, we take $\varepsilon$ to equal, $\varepsilon=1 /(4 \pi G)$, by analogy to electrostatics, where $G$ is Newton's constant. The gravitational susceptibility, $\chi$, is a measure of how gravitational dipoles respond to $\overrightarrow{g^{(0)}}$, where $\overrightarrow{g^{(0)}}$ is the external influence, the applied field. We will show later that cosmic susceptibility, $\bar{\chi}=\bar{\chi}(a)$, is epoch dependent, and possibly, $\varepsilon$, as well, if $G$ varies. Both are inherent properties of the vacuum. Locally, $\chi=\chi(\vec{x})$, is somewhat more complicated, in that the value of the gravitational field, the dipole moment, and the ambient temperature have to be specified. The local $\chi$ does not depend on cosmic conditions, but rather on local conditions, which is more involved. Given a net polarization, $\vec{P}(\vec{x})$, which is unequal to zero, we can proceed to find the average dipole moments, $\left\langle\overrightarrow{p_{d}}\right\rangle$, and the dipole number density. The gravitational dipole is defined as, $\overrightarrow{p_{d}} \equiv M_{P l} \vec{d}$, where $\vec{d}$ is the displacement vector, pointing from the negative mass planckion, to its nearest neighbor positive mass planckion. This definition is by analogy to electrostatics. It is known, namely, that,

$$
\vec{P}=\varepsilon \chi \vec{g}=n \overrightarrow{p_{d}}=n_{M A X}\left\langle\overrightarrow{p_{d}}\right\rangle
$$

In this equation, $n(\vec{x})$ is the effective dipole number density, and $n_{\text {MAX }}(\vec{x})$ equals the maximum dipole number density, in a region of space. The brackets, \langle\rangle , denote some sort of thermal average. The point is that $\overrightarrow{g^{(0)}}$ will lead to a possible polarization of the surrounding space, if conditions are right, and the ambient temperature is not too extreme. Local polarization can happen at very large ambient temperatures, provided the corresponding gravitational fields are substantial, as well. Cosmic susceptibility, on the other hand, being a smeared or average value for the universe as a whole, already is very weak at, 3000 Kelvin, since for the cosmos in total, the gravitational field is weak when averaged out.

Moreover, the polarization cloud produced by $\overline{g^{(0)}}$ will lead to bound mass density, $\rho_{B}$, which is induced through the formation of net dipole moments within the vacuum. We will identify, $\rho_{B}$, later with dark matter. Dark matter can exist locally, even at very high temperatures, such as those found in the bullet cluster, where the gravitational fields are large when compared to deep outer space. In regions where the gravitational field is not as large, such as deep interstellar space, dark matter can only survive at much reduced temperatures.

We have seen how the positive and negative mass planckions are anchored in position by means of fluid forces. It is now time to consider what macroscopic mass will do to the individual planckions. We postulate that free, or source, mass, made up of ordinary matter (like the earth), will attract positive mass planckions, and repel negative mass planckions. The reason we assume this has to do with anti-screening.

Consider a source mass, $M_{F}$, where, for simplicity, we assume spherical symmetry. This mass, $M_{F}$, will form a polarization cloud around it in the surrounding space, if conditions are right. The polarization is induced by the gravi- 
tational field, $\overrightarrow{g^{(0)}}$, which falls off as $1 / r^{2}$. We want polarization to point radially inwards, which means that, $\left\langle\overrightarrow{p_{d}}\right\rangle$, and, $\vec{P}$ point in the same direction as, $\overrightarrow{g^{(0)}}$. This will reinforce the original $g^{(0)}$ to create a macroscopic gravitational field, $\vec{g}=\overrightarrow{g^{(0)}}+\overrightarrow{g^{(1)}}$, which is greater than $\overrightarrow{g^{(0)}}$. The polarization, $\vec{P}$, is related to $\overrightarrow{g^{(1)}}$ by means of the equation, $\vec{P}=\varepsilon \chi \vec{g}=\varepsilon \overrightarrow{g^{(1)}}$. For $\overrightarrow{g^{(1)}}$ to point in the same direction as, $\overline{g^{(0)}}$, and produce anti-screening, we require that $M_{F}$ attracts the positive mass planckions, and repels the negative mass planckions. In other words, the positive planckion particles get displaced slightly radially inwards, whereas the negative planckion particles get pushed slightly radially outwards. This creates the dipole, which can be aligned or ordered if ambient temperatures are sufficiently low. A larger $\vec{g}$ versus $\overline{g^{(0)}}$ value is needed for explaining dark matter. Anti-screening will be discussed in greater detail in the next section.

\section{Gravi-Statics and Gravitational Polarization}

In this section, we present a theory of polarization as it would apply to gravitational fields. We proceed by analogy to electrostatics, which is familiar. There will be some notable differences.

We start by defining the following gravitational fields. Let, $\overrightarrow{g^{(0)}} \equiv$ the gravitational field produced by macroscopic mass, i.e., aggregate matter such as gas, dust, planets, stars, galaxies, etc. In general, $\overrightarrow{g^{(0)}}(\vec{x})$, is a local field. When dealing with the cosmos as a whole, however, $\overline{g^{(0)}}$ is a smeared or average quantity, valid only if huge distance scales are considered, in excess of, $100 \mathrm{Mpc}$.

$\overrightarrow{g^{(1)}} \equiv$ the induced gravitational field due to gravitational dipole response, and ordering. In general, $\overrightarrow{g^{(1)}}(\vec{x})$, is a local field. When dealing with the cosmos as a whole, however, $\overline{g^{(1)}}$ is a smeared or average quantity, valid only if huge distance scales are considered, in excess of, $100 \mathrm{Mpc}$.

$\vec{g} \equiv \overrightarrow{g^{(0)}}+\overrightarrow{g^{(1)}}=$ the total macroscopic gravitational field, as measured in space, which takes the "gravitic" into account. The gravitic is our gravitational version of a dielectric, and represents the vacuum filling the cosmos. The gravitic is comprised of positive and negative mass planckions in our model. In general, $\vec{g}(\vec{x})$, is a local field. When dealing with the cosmos as a whole, however, $\bar{g}$ is a smeared or average quantity, valid only if huge distance scales are considered, in excess of, $100 \mathrm{Mpc}$.

$\overrightarrow{g^{(2)}} \equiv$ the gravitational field experienced by an individual dipole within the gravitic. In electro-statics, this is sometimes referred to as the local Lorentz field, or "molecular field". In general, $\overrightarrow{g^{(2)}}(\vec{x})$, is a local field. This takes into account the effects of the other dipoles in the vicinity of the dipole in question. $\overrightarrow{g^{(2)}}(\vec{x})$ is typically proportional to $\vec{g}(\vec{x})$, and depends on the lattice symmetry, and the local susceptibility, $\chi(\vec{x})$. We will use, $\overrightarrow{g^{(2)}}(\vec{x})$, in later work, but specify it here for completeness.

$(3-1 a, b, c, d)$

All quantities with a bar over them are smeared or average values, which, by 
definition, are non-local. When considered on a grand scale, the universe is only homogeneous in density, and spherically symmetric, if distance scales in excess of about $100 \mathrm{Mpc}$ are considered. If $\bar{\chi}$ is the susceptibility of space, then $\overline{g^{(1)}}=\bar{\chi} \bar{g}$, and the polarization can be written as, $\bar{P}=\varepsilon \bar{\chi} \bar{g}=\varepsilon \overline{g^{(1)}}$. As mentioned before, the gravitational permittivity, $\varepsilon$, is defined by the equation, $\varepsilon \equiv 1 /(4 \pi G)$, where $G$ is Newton's constant. Since we are considering the present epoch only in this paper, we could just as well replace, $\varepsilon$, by, $\varepsilon=\varepsilon_{0} \equiv 1 /\left(4 \pi G_{0}\right)=1.192 \mathrm{E} 9(\mathrm{MKS})$.

The susceptibility, $\chi$, is not in general a constant, but rather a non-linear function, which depends on the value of dipole moment, gravitational field, and ambient temperature. In this instance, it is a local variable, a field. When dealing with the cosmos as a whole, however, $\bar{\chi}$, is a smeared or average quantity, and it will depend only on " $a$ ", the cosmic scale parameter, i.e., $\bar{\chi}=\bar{\chi}(a)$. Within a cosmological era, it will therefore have a fixed value. In this paper, we will be dealing exclusively with $\bar{\chi}=\bar{\chi}\left(a_{0}=1\right)$.

In electrostatics, the induced electric field, $\overrightarrow{E^{(1)}}$, takes away from the source, or applied, electric field, $\overline{E^{(0)}}$. This is called screening. Here, in gravistatics, the induced gravitational field, $\overrightarrow{g^{(1)}}$, will add to the applied field, $\overrightarrow{g^{(0)}}$, which signifies anti-screening. This is best seen by the following picture. In a small enough region of space, the applied field is relatively constant in both magnitude and direction. Let that applied field be due to a sheet of uniform positive charge for electrostatics, and a sheet of uniform positive mass, for gravistatics. We position both sheets to the left. In electrostatics, the electric field, $\overrightarrow{E^{(0)}}$, is pointing to the right, in the positive $\hat{X}$ direction, whereas in gravistatics, the gravitational field, $\overrightarrow{g^{(0)}}$, points to the left, in the negative $\hat{x}$ direction. If we insert a dielectric in the electric field case, then a polarization will result if conditions are right, and that polarization will point from negative to positive charge within the dielectric, i.e., in the positive $\hat{X}$ direction. In the gravitational field case, we insert a gravitic, the gravitational equivalent of a dielectric. In the applied gravitational field, the polarization will point to the left, in the negative $\hat{x}$ direction, since the positive mass planckions are attracted to the sheet, and the negative mass planckions are repulsed by it. In the gravitational field case, this polarization will reinforce the original field, $\overrightarrow{g^{(0)}}$, since, $\vec{P}=\varepsilon \overrightarrow{g^{(1)}}$, also points in the same direction. Hence, $\vec{g}=\overrightarrow{g^{(0)}}+\overrightarrow{g^{(1)}}>\overrightarrow{g^{(0)}}$. In the electric field case, we have the opposite effect. There, the induced field opposes the applied field, and we have, $\vec{E}=\overrightarrow{E^{(0)}}+\overrightarrow{E^{(1)}}<\overrightarrow{E^{(0)}}$.

Mathematically, in the gravistatic situation, we therefore have,

$$
\vec{g}=\overrightarrow{g^{(0)}}+\overrightarrow{g^{(1)}}=(K+\chi) \vec{g}=\vec{g}
$$

By contrast, in the electrostatic situation, we obtain,

$$
\vec{E}=\overrightarrow{E^{(0)}}+\overrightarrow{E^{(1)}}=(K-\chi) \vec{E}=\vec{E}
$$

within the dielectric, $E=E^{(0)} / K=E^{(0)} /(1+\chi)<E^{(0)}$, whereas, in the gravitic (or cosmic vacuum), $g=g^{(0)} / K=g^{(0)} /(1-\chi)>g^{(0)}$. The, $\chi$, and $K$, are the 
susceptibility, and relative permittivity, respectively, for each situation. The gravitic is, according to our hypothesis, made up of the positive and negative mass planckions, which form the vacuum.

The induced fields, whether they are electric or gravitational in nature, will always reflect the symmetry of the underlying source, whether that symmetry be spherical, cylindrical, rectangular, etc. Thus, for example, if, $\overrightarrow{g^{(0)}}$, in Equation (3-2), displays cylindrical symmetry, then so too, will, $\overrightarrow{g^{(1)}}$, and $\vec{g}$, which is the sum of both $\overline{g^{(0)}}$, and $\overrightarrow{g^{(1)}}$.

As mentioned, the gravitational fields, defined in (3-1a, b, c), need not be local fields. They could be an average or smeared quantity, as when dealing with the universe as a whole. In a given epoch, the cosmic, $\overline{g^{(0)}}, \overline{g^{(1)}}$ and $\bar{g}$ are all smeared or average values, which hold for the universe as a whole. If huge distance scales are considered, then, $\overline{g^{(0)}}=G \sum_{i=1}^{N}\left(M_{F, i} / r_{i}^{2}\right)=G M_{F} / R^{2}$. The sum is over all stars, galaxies, planets, gases, dust, etc. contained within a very large radius, $R$, greater than about $100 \mathrm{Mpc}$. A smeared quantity will not scale according to the equation above, upon expansion of space. Rather a different scaling law will apply, to be specified in a follow-up paper.

We can now develop the relations needed. We start with free, or source mass, i.e., all ordinary matter in the universe made up of quarks and leptons. We apply Gauss law to such a mass, and find,

$$
-\oint \overrightarrow{g^{(0)}} \cdot \mathrm{d} \vec{A}=1 / \varepsilon \sum M_{i}=1 / \varepsilon M_{F}
$$

As always, $\varepsilon \equiv 1 /(4 \pi G)$. The gravitational permittivity, $\varepsilon$, may or may not be a constant, depending on whether $G$ is variable. If $G$ varies cosmologically, then, $G=G(a)$, and $\varepsilon=\varepsilon(a)$. In the present epoch, however, the $G$ value is definitely fixed, and we can replace $\varepsilon$ by, $\varepsilon_{0}=1.192 \mathrm{E} 9$ (MKS). The present paper deals, principally, with the current era. We can bring the $\varepsilon$ over to the left hand side, and place it within the integral as this is a surface integral. If $G$ were to vary cosmologically, and this was a volume integral, we could not do this. But because we are dealing with a surface, and $\varepsilon(a)$ has a constant value everywhere on the surface, this is allowed, whether, or not, $G$ is a constant. Doing this we obtain,

$$
-\oint \varepsilon \overrightarrow{g^{(0)}} \cdot \mathrm{d} \vec{A}=\sum M_{i}=M_{F}
$$

We next use Stokes theorem, and convert this to a volume integral.

$$
-\int \vec{\nabla} \cdot\left(\varepsilon \overrightarrow{g^{(0)}}\right) \mathrm{d} V=\int \rho_{F} \mathrm{~d} V
$$

From this, it follows that the divergence of, $\left(\varepsilon \overrightarrow{g^{(0)}}\right)$, equals,

$$
-\vec{\nabla} \cdot\left(\varepsilon \overrightarrow{g^{(0)}}\right)=\rho_{F}=\overrightarrow{-\nabla} \cdot \vec{D} \text { where, } \vec{D} \equiv \varepsilon \overrightarrow{g^{(0)}}
$$

The vector, $\vec{D}$, defined in Equation (3-7), is called the displacement field in electrostatics and we use the same terminology here for its gravitational counterpart. We note that $\vec{D}$ can also be written as, $\vec{D} \equiv \varepsilon \overrightarrow{g^{(0)}}=\varepsilon K \vec{g}$. See Equa- 
tion (3-2). Wherever we have a non-zero gravitational mass distribution, $\rho_{F}(\vec{x})$, we can expect a gravitational displacement field, $\vec{D}(\vec{x})$.

Furthermore, for spherically symmetric mass distributions, the divergence of $\vec{D}$ becomes,

$$
1 / r^{2} \mathrm{~d}\left(r^{2} \varepsilon g^{(0)}\right) / \mathrm{d} r=\rho_{F}
$$

where now, $\rho_{F}=\rho_{F}(r)$. We consider the radius of the Hubble universe, and let, $r=R$. In this instance, Equation (3-8), can be re-expressed as,

$$
4 \pi d\left(R^{2} \varepsilon g^{(0)}\right)=4 \pi \rho_{F} R^{2} \mathrm{~d} R
$$

Upon integration, this gives the very familiar expression,

$$
g^{(0)}=G M_{F} / R^{2}
$$

We note that $\overrightarrow{g^{(0)}}$ points radially inwards, and Equation (3-10), holds true irrespective of whether, or not, $G$ is a constant. The mass, $M_{F}$, is the total, ordinary mass contained within the Hubble radius; it will remain constant as the radius expands. The value of, $g^{(0)}$, in Equation (3-10), is actually a smeared or average value, and should properly be designated with a bar over it, as in, $g^{(0)}=\overline{g^{(0)}}$.

Another way to express Equation (3-10), is to use the mass-density relation. Then,

$$
\overline{g^{(0)}}=G \bar{\rho}_{F}(4 \pi / 3) R=\bar{\rho}_{F} R /(3 \varepsilon)
$$

In this equation, $\bar{\rho}_{F}$ represents the smeared mass density within radius, $R$, the Hubble radius, due to all the ordinary mass contained within it. The $\bar{\rho}_{F}$ value is fairly constant for distance scales greater than $100 \mathrm{Mpc}$ (current epoch).

We next generalize Gauss law to include bound mass, $M_{B}$, as well as free, source mass. Bound mass is that due to the polarization field, and the induced dipole moments, which are ordered or organized within a region of space. Those dipole moments are gravitational. We have,

$$
-\oint \varepsilon \vec{g} \cdot \mathrm{d} \vec{A}=-\int \vec{\nabla} \cdot(\varepsilon \vec{g}) \mathrm{d} V=M_{F}+M_{B}
$$

And, because, $\vec{g}=\overrightarrow{g^{(0)}}+\overrightarrow{g^{(1)}}$, by Equation (3-2), we can further decompose this into its component parts as,

$$
\begin{aligned}
& -\oint \varepsilon \overrightarrow{g^{(0)}} \cdot \mathrm{d} \vec{A}=-\int \vec{\nabla} \cdot\left(\varepsilon \overrightarrow{g^{(0)}}\right) \mathrm{d} V=M_{F}=\int \rho_{F} \mathrm{~d} V=-\int \vec{\nabla} \cdot \vec{D} \mathrm{~d} V \\
& -\oint \varepsilon \overrightarrow{g^{(1)}} \cdot \mathrm{d} \vec{A}=-\int \vec{\nabla} \cdot\left(\varepsilon \overrightarrow{g^{(1)}}\right) \mathrm{d} V=M_{B}=\int \rho_{B} \mathrm{~d} V=-\int \vec{\nabla} \cdot \vec{P} \mathrm{~d} V
\end{aligned}
$$

From both these equations, it is obvious that,

$$
-\vec{\nabla} \cdot \vec{D}=\rho_{F} \quad \text { and }-\vec{\nabla} \cdot \vec{P}=\rho_{B}
$$

The $\vec{P}=\varepsilon \overrightarrow{g^{(1)}}$, is, of course, the polarization field set up within the vacuum, the gravitic medium, made up of positive and negative mass planckions. These planckions have organized themselves into gravitational dipoles, which do not vanish macroscopically. 
In an applied field, $\overrightarrow{g^{(0)}}$, we can have ionic polarization, where the positive and negative masses are displaced slightly to form the dipole. This dipole is induced. We can also have orientation polarization, where permanent dipoles are aligned in the direction of the source, or applied gravitational field. The a-priori permanent dipoles could be present due to CBR blackbody photon bombardment, and what remains is for the dipole axis to align itself in the direction of the source field. Either way, a net polarization can result, if conditions are right. There are other forms of polarization possible, but these are good starting points.

Equations (3-12), (3-13), and (3-14), allow us to write,

$$
\vec{\nabla} \cdot(\varepsilon \vec{g})=\vec{\nabla} \cdot \vec{D}+\vec{\nabla} \cdot \vec{P}
$$

Or, more simply,

$$
(\varepsilon \vec{g})=\vec{D}+\vec{P}
$$

Equation (3-17), is Equation (3-2), multiplied by, $\varepsilon=1 /(4 \pi G)$. Notice that in electrostatics, we have, in contrast to Equation (3-17), the relation, $\left(\varepsilon_{0} \vec{E}\right)=\vec{D}-\vec{P}$, with a negative sign. In electrostatics, the electric permittivity, equals,

$\varepsilon_{0}=1 /(4 \pi k)=8.85 \mathrm{E}-12(\mathrm{MKS})$. In Equations (3-16), and (3-17), however, we are using the gravitational permittivity, $\varepsilon=\varepsilon_{0}=1 /\left(4 \pi G_{0}\right)=1.192 \mathrm{E} 9$ (MKS)

Another way to write Equation (2-16), is

$$
-\vec{\nabla} \cdot(\varepsilon \vec{g})=\rho=\rho_{\text {TOTAL }}=\rho_{F}+\rho_{B}
$$

This follows using the relations, (15a, b). An equation such as, (3-18), is valid both locally, and cosmically, as a smeared quantity. As a local equation, we would have, $\rho(\vec{x})=\rho_{F}(\vec{x})+\rho_{B}(\vec{x})$. As a cosmic equation, where we have smeared or average values, this equation would read, $\bar{\rho}=\overline{\rho_{F}}+\overline{\rho_{B}}$. Many of the equations, but not all, in this paper can be viewed, and interpreted, both ways. The density parameters in the Friedmann equation are smeared or average values, holding for the universe as a whole. For cosmic quantities, the appropriate temperature is the CBR temperature, and the mass densities evolve epoch by epoch. Within a given cosmological era, smeared values are constant. Thus, $\bar{\chi}=\bar{\chi}(a)$ has a fixed value in this era, versus another fixed value in another era. We have seen that,

$$
\vec{P}=\varepsilon \chi \vec{g}=\varepsilon \overrightarrow{g^{(1)}},-\vec{\nabla} \cdot \vec{P}=\rho_{B}
$$

We likewise have,

$$
\vec{D}=\varepsilon K \vec{g}=\varepsilon \overrightarrow{g^{(0)}},-\vec{\nabla} \cdot \vec{D}=\rho_{F}
$$

If we combine both, we obtain,

$$
\varepsilon \vec{g}=\varepsilon(K+\chi) \vec{g},-\vec{\nabla} \cdot(\varepsilon \vec{g})=\rho=\rho_{F}+\rho_{B}
$$

In electrostatics, we have, $(K-\chi)=1$, which indicates screening. But in gravistatics, we notice that, $(K+\chi)=1$, which signifies anti-screening. See Equations (3-2), and (3-3), and the discussion around them. Some further useful 
relations are,

$$
\vec{P}=(\chi / K) \vec{D}, \overline{\rho_{B}}=(\chi / K) \overline{\rho_{F}}
$$

Both of these equations follow directly from relations, (3-19a, b), and (3-20a, b). Integrating Equation (3-22b), over a three-dimensional volume gives, $M_{B}=(\chi / K) M_{F}$.

For spherical symmetry, we have seen that Equation (3-10), is valid, where a bar should be placed over the $g^{(0)}$. It should come as no surprise then, that similar relations hold for, $\overline{g^{(1)}}$, and $\bar{g}$. We have,

$$
\overline{g^{(1)}}=G M_{B} / R^{2}, \bar{g}=G\left(M_{F}+M_{B}\right) / R^{2}
$$

The total mass contained within the Hubble bubble is, $M=M_{\text {TотАL }}=\left(M_{F}+M_{B}\right)$. Bound mass, $M_{B}$, is an inherent assumption in our model.

In the present epoch, we could attach subscripts " 0 " on all cosmological, i.e., smeared quantities. The free mass value, $M_{F}$, will not change in transitioning between epochs, provided we do not go further back in time than BBN (Big Bang Nucleosynthesis). The bound mass, however, will change because of the relation, $M_{B}=(\chi / K) M_{F}$. Both the cosmic susceptibility, $\chi=\chi(a)$, and, the cosmic relative permittivity, $K=K(a)=1-\chi(a)$, will change if we transition between epochs. As the CBR temperature cools upon expansion of the universe, we expect $\chi(a)$ to become larger, and, $K(a)$, correspondingly, weaker in value. We give two specific functions for $\chi(a)$ in a follow up paper. The $\overline{\rho_{F}}$ value in Equation (3-22b), is roughly the equivalent of 6 hydrogen atoms per cubic meter.

Every observer within the universe, irrespective of location, will measure the same magnitude for, $\overline{g^{(0)}}$, given by Equation (3-10). The direction will be different, as $\overrightarrow{g^{(0)}}$ will always point radially inwards, towards the observer. But the magnitude will be the same because the $G$ value, the $M_{F}$ value, and the $R$ value will not differ in going from observer to observer in the cosmos. The radius, $R$, refers to the Hubble radius. Technically, $\overrightarrow{g^{(0)}}$, is a surface integral defined over a Gaussian surface, but this surface gravitational field holds point for point within the cosmos, as no one spot in the universe is preferable over another.

Another way of arguing this point is to recognize that observer, A, would measure the value, $\overline{g_{A}^{(0)}}$, across his/her Hubble bubble. A different observer, B, would measure, $\overline{g_{B}^{(0)}}$, across their bubble. At the points of intersection of the two bubbles, we would have, $\overline{g_{A}^{(0)}}=\overline{g_{B}^{(0)}}$. This value would extend through both surfaces of observers, A, and B. Since point for point within the universe, we have intersecting Hubble surfaces, it follows that, $\overline{g_{A}^{(0)}}=\overline{g_{B}^{(0)}}=\overline{g^{(0)}}$. A third way of arguing is that one observer's surface is another observer's center. Therefore, what one observer measures, is directly applicable and can be carried over, to the second observer's location. The universe is made up of intersecting bubbles, all with the same $\overline{g^{(0)}}$ value. Summarizing, $\overline{g^{(0)}}$ definitely exists, and will have the same value, as one moves from location to location, within the cosmos. The, $\overline{g^{(0)}}$, will lead to terms in Friedmann's equation which cannot be ignored. 
We close this section with a table, Table 1 . This table summarizes some of the similarities, and some of the notable differences between electrostatics, and gravistatics. This can be used as a quick reference guide. The $Q$ represents charge in electrostatics.

\section{Interpretation of Dark Matter and Dark Energy Terms in the Friedmann Equation}

In this section, we provide a model for the density parameters associated with the Friedmann equation. As is well-known, the Friedmann equation can be written in the form,

$$
\begin{aligned}
H^{2} & =8 \pi G / 3\left(\rho_{\text {Radiaion }}+\rho_{O M}+\rho_{D M}+\rho_{\Lambda}\right) \\
& =8 \pi G / 3\left(\Omega_{\text {Radiaion }}+\Omega_{O M}+\Omega_{D M}+\Omega_{\Lambda}\right) \rho \\
& =8 \pi G / 3 \rho
\end{aligned}
$$

\begin{tabular}{|c|c|}
\hline Electrostatics & Gravistatics \\
\hline Like charges repel & Like planckion masses repel and attract, \\
\hline \multirow[t]{2}{*}{ Unlike charges attract } & Unlike planckion masses attract and repel, indirectly \\
\hline & $\begin{array}{l}\text { Fluid forces acting on each species, the positive, } \\
\text { and the negative mass planckions }\end{array}$ \\
\hline$\vec{E}=\overrightarrow{E^{(0)}}-\overrightarrow{E^{(1)}}$ & $\vec{g}=\overrightarrow{g^{(0)}}+\overrightarrow{g^{(1)}}$ \\
\hline$=(K-\chi) \vec{E}$ & $=(K+\chi) \vec{g}$ \\
\hline$\vec{E}=\overrightarrow{E^{(0)}} / K<\overrightarrow{E^{(0)}} \quad$ (screening) & $\vec{g}=\overrightarrow{g^{(0)}} / K>\overrightarrow{g^{(0)}} \quad$ (anti-screening) \\
\hline$\vec{P}=\varepsilon_{0} \chi \vec{E}=\varepsilon_{0} \overrightarrow{E^{(1)}}$ & $\vec{P}=\varepsilon_{0} \chi \vec{g}=\varepsilon_{0} \overrightarrow{g^{(1)}}$ \\
\hline$k=1 /\left(4 \pi \varepsilon_{0}\right)=8.99 \mathrm{E} 9(\mathrm{MKS})$ & $G_{0}=1 /\left(4 \pi \varepsilon_{0}\right)=6.67 \mathrm{E}-11(\mathrm{MKS})$ \\
\hline$\vec{\nabla} \cdot \vec{P}=\rho_{B}$ & $-\vec{\nabla} \cdot \vec{P}=\rho_{B}$ \\
\hline$\oint \vec{P} \cdot \overrightarrow{\mathrm{d} A}=Q_{B}$ & $-\oint \vec{P} \cdot \overrightarrow{\mathrm{d} A}=M_{B}$ \\
\hline$\vec{D}=\varepsilon_{0} K \vec{E}=\varepsilon_{0} \overrightarrow{E^{(0)}}$ & $\vec{D}=\varepsilon_{0} K \vec{g}=\varepsilon_{0} \overrightarrow{g^{(0)}}$ \\
\hline$\vec{\nabla} \cdot \vec{D}=\rho_{F}$ & $-\vec{\nabla} \cdot \vec{D}=\rho_{F}$ \\
\hline$\oint \vec{D} \cdot \overrightarrow{\mathrm{d} A}=Q_{F}$ & $-\oint \vec{D} \cdot \overrightarrow{\mathrm{d} A}=M_{F}$ \\
\hline$\vec{\nabla} \cdot\left(\varepsilon_{0} \vec{E}\right)=\rho_{F}-\rho_{B}$ & $-\vec{\nabla} \cdot\left(\varepsilon_{0} \vec{g}\right)=\rho_{F}+\rho_{B}$ \\
\hline$\oint\left(\varepsilon_{0} \vec{E}\right) \cdot \overrightarrow{\mathrm{d} A}=Q_{F}-Q_{B}$ & $-\oint\left(\varepsilon_{0} \vec{g}\right) \cdot \overrightarrow{\mathrm{d} A}=M_{F}+M_{B}$ \\
\hline$\vec{E}=\overrightarrow{E^{(0)}}-\overrightarrow{E^{(1)}}$ & $\vec{g}=\overrightarrow{g^{(0)}}+\overrightarrow{g^{(1)}}$ \\
\hline can be written as, & can be written as, \\
\hline$\left(\varepsilon_{0} \vec{E}\right)=\vec{D}-\vec{P}$ & $\left(\varepsilon_{0} \vec{g}\right)=\vec{D}+\vec{P}$ \\
\hline$\vec{D}=$ electric displacement field & $\vec{D}=$ gravitational displacement field \\
\hline$Q=Q_{\text {TOTAL }}=Q_{F}-Q_{B}$ & $M=M_{\text {TOTAL }}=M_{F}+M_{B}$ \\
\hline$\rho=\rho_{\text {TOTAL }}=\rho_{F}-\rho_{B}$ & $\rho=\rho_{\text {тотAL }}=\rho_{F}+\rho_{B}$ \\
\hline
\end{tabular}

Table 1. Some similarities and some notable differences between electrostatics and planckion gravistatics. 
In Equation (4-1), $G$ is Newton's constant, and, $\rho$, is the total mass density. In the present epoch, the total mass density equals, $\rho_{0}=8.624 \mathrm{E}-27 \mathrm{~kg} / \mathrm{m}^{3}$, corresponding to a Hubble rate of expansion of, $H_{0}=67.74 \mathrm{~km} /(\mathrm{s} \cdot \mathrm{Mpc})$. All $\Lambda \mathrm{CDM}$ parameters are taken from the 2016 Planck XIII cosmological parameter collaboration (final release) [3]. The component mass densities,

$\rho_{\text {Radiaion }}, \rho_{O M}, \rho_{D M}, \rho_{\Lambda}$, are the radiation, ordinary matter, dark matter, and dark energy contributions, respectively. In terms of their relative weightings, we have, $\left(\Omega_{\text {Radiaion }}, \Omega_{O M}, \Omega_{D M}, \Omega_{\Lambda}\right)=(9.153 \mathrm{E}-5,0.0486,0.2589,0.6911)$. The sum of the density parameters, $\sum \Omega_{i}$, should equal unity, since all indications are that the universe is flat. The sum above is slightly less than one because we have ignored certain terms such as electrons, and other minor contributions, to the overall density.

Gravitational blackbody radiation has been considered by various authors [27] [28] [29], and when compared to photon and neutrino contributions, thought to be negligible. The value for, $\Omega_{\text {Radiation }}=9.153 \mathrm{E}-5$, given above, is due to photons and neutrinos only. The freeze-out temperature for graviton blackbody radiation is calculated to lie somewhere in the range, 1 - 1.6 degrees Kelvin, less than the 1.9 degree Kelvin freeze-out temperature for neutrinos. We also only have one flavor, and therefore the correction to, $9.153 \mathrm{E}-5$, is believed to be minor. The estimates are, $3 \mathrm{E}-33 \mathrm{~kg} / \mathrm{m}^{3}$ or, $\Omega_{\text {R Radiation }}=3.48 \mathrm{E}-7$ for, 1 Kelvin. And for, 1.6 Kelvin, the number is closer to, $2 \mathrm{E}-32 \mathrm{~kg} / \mathrm{m}^{3}$ or,

$\Omega_{g \text { Radiation }}=2.32 \mathrm{E}-6$. This correction will be ignored, since it is so small.

Under ordinary matter density, $\rho_{O M}$, we should also, more properly, include electrons, and massive neutrinos, which has not been done. The masses for the neutrinos are uncertain, and the electrons are relatively minor corrections as well. These have been ignored in setting, $\rho_{O M}=0.0486$. The value, 0.0486 , really only refers to the baryonic mass, and hence, it is often written as, $\Omega_{b}=0.0486$. We will work with the $\Omega_{b}=0.0486$ value, as this is the value, which is most often quoted. Under ordinary matter, we understand matter made up of quarks and leptons, held together by strong and electromagnetics forces, found in the universe at large, in luminous and nonluminous form. This includes plasma particles, simple atoms and molecules, interstellar gas, dust, planets, stars, galaxies, etc.

From the $\Lambda$ CDM model, we have, $\Omega_{b} h^{2}=0.02230$. Since, $h=0.6774$, this translates into $\Omega_{b}=0.0486$. For dark matter, $\Lambda$ CDM gives, $\Omega_{c} h^{2}=0.1189$. Thus, $\Omega_{c}=\Omega_{D M}=0.2589$. This component is poorly understood, as is the dark energy contribution, $\Omega_{\Lambda}=\Omega_{D E}=0.6911$. We are working exclusively in the present epoch in this paper, and the subscript, "0", on all variables will, by and large, be dropped.

We next introduce, in place of Equation (4-1), a second very similar looking equation. This equation has the form,

$$
\begin{aligned}
H^{2} & =8 \pi G / 3\left(\rho_{\text {Radiaion }}+\rho_{F}+\rho_{B}+\rho_{g g}\right) \\
& =8 \pi G / 3\left(\Omega_{\text {Radiaion }}+\Omega_{F}+\Omega_{B}+\Omega_{g g}\right) \rho
\end{aligned}
$$


For our purposes, we identify and set, $\Omega_{F}=0.0486, \Omega_{B}=\Omega_{D M}=0.2589$, and, $\Omega_{g g}=\Omega_{D E}=0.6911$. The density parameter, $\Omega_{F}$, refers to all the ordinary mass in the universe, baryonic and leptonic. This is what we sometimes call "free" mass, or "source" mass. The density parameter, $\Omega_{B}$, is bound mass induced by free mass, due to polarization of the surrounding space, and identified by us as dark matter, $\Omega_{c}$. And the, $\Omega_{g g}$, is our version of dark energy, where, $\Omega_{g g}$, is set equal to, $\Omega_{\Lambda}=\Omega_{D E}=0.6911$. It is due to the gravitational fields associated with both source matter, and bound matter. Source matter, and bound matter, both have a net gravitational field, which cannot be ignored in Friedmann's equation. By Gauss law these gravitational fields are very real because $\overline{\rho_{F}}$ definitely exists throughout the cosmos, and we assume that, $\overline{\rho_{B}}$, exists as well, if we believe in polarization.

Normally, gravitational fields have a mass density, which is insignificant when compared to source mass density. This is certainly the case for the earth, where the average mass density of the earth equals, $\overline{\rho_{F}}=5.51 \mathrm{E} 3 \mathrm{~kg} / \mathrm{m}^{3}$. Keep in mind, however, that on the earths' surface, we also have an additional gravitational field in the amount of, $\overline{g^{(0)}}=9.81 \mathrm{~m} / \mathrm{s}^{2}$, and this will lead to a mass density, in its own right, of, $6.37 \mathrm{E}-7 \mathrm{~kg} / \mathrm{m}^{3}$. When compared with the $\overline{\rho_{F}}$ value above, we notice that it can safely be ignored. However, when dealing with a very dilute universe, the tables are turned. It will actually turn out that $\Omega_{g g}$ is greater than either, $\Omega_{F}$, or, $\Omega_{B}$. There is more energy density associated with gravitational fields in the universe, than anything else, if our thinking is correct.

Following electrostatics, we set, $\rho_{g g}$, in Equation (4-2), equal to,

$$
\begin{aligned}
\rho_{g g} & =1 /\left(2 c^{2}\right) K \varepsilon g^{2} \\
& =\Omega_{g g} \rho \\
& =1 /\left(2 c^{2}\right) \varepsilon g g^{(0)} \\
& =1 /\left(2 c^{2}\right) \varepsilon g^{(0)}\left(g^{(0)}+g^{(1)}\right) \\
& =1 /\left(2 c^{2}\right) \varepsilon g^{(0)} g^{(0)}+1 /\left(2 c^{2}\right) \varepsilon g^{(0)} g^{(1)}
\end{aligned}
$$

We are using the notation of the last section. In Equation (4-3), $K$ is the relative permittivity, but now for the gravitic medium, the vacuum, made up of positive and negative mass planckions. The gravitational permittivity has the value, $\varepsilon \equiv 1 /(4 \pi G)$, which in the present epoch, amounts to, $\varepsilon=\varepsilon_{0} \equiv 1 /\left(4 \pi G_{0}\right)=1.192 \mathrm{E} 9$ (MKS). The, $G_{0}$, stands for Newton's constant, $G_{0}=6.674 \mathrm{E}-11(\mathrm{MKS})$, which is the only $G$ value considered in this paper.

We notice in Equation (4-3), that the macroscopic gravitational field is made up of two components, $g^{(0)}=K g$, and, $g^{(1)}=\chi g$, using the notation of the last section. The $g^{(0)}$ is the gravitational field due strictly to source matter, or ordinary matter, whereas, $g^{(1)}$, refers to the gravitational field set up by polarized matter, the vacuum's response to the external field. In the last line of Equation (4-3), we have a coupling of the source gravitational field with itself, but also a second term, where the source gravitational field couples to the polarization 
gravitational field, i.e., the gravitational field set up within the vacuum.

Thus, we can decompose $\rho_{g g}$ into two separate parts,

$$
\rho_{g g}=\rho_{A A}+\rho_{B B}
$$

where,

$$
\begin{aligned}
& \rho_{A A} \equiv 1 /\left(2 c^{2}\right) \varepsilon g^{(0)} g^{(0)} \\
& \rho_{A B} \equiv 1 /\left(2 c^{2}\right) \varepsilon g^{(0)} g^{(1)}
\end{aligned}
$$

Equations (4-5a, b), are formal definitions. Since we are dealing with the current epoch, the radiation contribution to mass density, $\rho_{\text {Radiation }}$, will be ignored. Relative to the other terms, this contribution is negligible. We focus on ordinary matter, dark matter, and dark energy,

In electrostatics, the energy density associated with the electric field, in the presence of a dielectric, is, $u_{E E}=1 / 2 \vec{D} \cdot \vec{E}=1 / 2 K \varepsilon_{0} E^{2}$, where $\vec{D}$ is the electric displacement field, $K$, the relative electric permittivity, and, $\varepsilon_{0}$, equals the electric permittivity of free space. In gravistatics, we must have something analogous, and therefore, we write, $u_{g g}=1 / 2 \vec{D} \cdot \vec{g}=1 / 2 K \varepsilon_{0} g^{2}$, where $\vec{g}$ is the gravitational field, $\vec{D}$ is the gravitational displacement field, and $K$, the relative gravitational permittivity. Also, $\varepsilon_{0}$, equals the gravitational permittivity of the vacuum, defined as. $\varepsilon_{0} \equiv 1 /\left(4 \pi G_{0}\right)=1.192 \mathrm{E} 9(\mathrm{MKS})$. If we define, $\rho_{g g}$, as, $\rho_{g g} \equiv u_{g g} / c^{2}$, we obtain our equation for gravitational field mass density, (4-3), above.

We emphasize once more that in electrostatics, the relative permittivity, $K=1+\chi$, is greater than unity, which produces screening. In gravistatics, by contrast, $K=1-\chi$, is less than one, which gives anti-screening. In other words, $\vec{g}=\overrightarrow{g^{(0)}}+\overrightarrow{g^{(1)}}>\overrightarrow{g^{(0)}}$, whereas, $\vec{E}=\overrightarrow{E^{(0)}}-\overrightarrow{E^{(1)}}<\overrightarrow{E^{(0)}}$. The $\chi$ in gravistatics is the cosmic susceptibility, satisfying, $\bar{P}=\varepsilon_{0} \chi \bar{g}=\varepsilon_{0} g^{(1)}$, where $\bar{P}$ is the smeared polarization of space, when considered on grand scale. Both, $\chi$, and $K=1-\chi$, are thought to be epoch dependent, if defined as a cosmic average. In other words, $\chi=\chi(a)$, where, $a$, is the cosmic scale parameter. Two specific functions (models) for the cosmic $\chi(a)$ will be presented in a follow-up paper.

Coming back to Equations (4-2), and (4-4), we have made the following identifications.

$$
\begin{aligned}
& \rho_{F}=\rho_{O M} \cong \rho_{b}=0.0486 \rho_{0} \\
& \rho_{B}=\rho_{D M}=\rho_{c}=0.2589 \rho_{0} \\
& \rho_{g g}=\rho_{D E}=\rho_{\Lambda}=0.6911 \rho_{0}
\end{aligned}
$$

Different ways to specify ordinary matter, and dark matter, respectively, in Friedmann's equation, are

$$
\rho_{F}=-\vec{\nabla} \cdot \vec{D}, \quad \rho_{B}=-\vec{\nabla} \cdot \vec{P}
$$

These equations are cosmic averages for a smeared universe. We have used the results from the previous section.

We first work with Equations (4-6a), and (4-6b). Setting up a ratio, we find 
that

$$
\rho_{B} / \rho_{F}=0.2589 / 0.0486=5.327
$$

However, from the previous section, it is also known that,

$$
\rho_{B} / \rho_{F}=-\vec{\nabla} \cdot \vec{P} /(-\vec{\nabla} \cdot \vec{D})=\chi_{0} / K_{0}
$$

See Equation (3-24b), and Equation (3-21b), with, (3-22b). We have included the subscript on, $\chi_{0}$, and $K_{0}$ in Equation (4-9), to emphasize that we are working in the present epoch. Also, technically, we should have bars over the various densities in the above equations, as well as over the, $\chi_{0}$, and $K_{0}$ variables, as we are dealing with smeared or average values for the cosmos as a whole. We dispense with that, in order to simplify the notation.

We next compare Equation (4-9), with Equation (4-8). They are the same, and therefore, it follows that

$$
\chi_{0} / K_{0}=5.327
$$

This is easily solved since, $K_{0}=1-\chi_{0}$. The solutions are,

$$
\chi_{0}=0.842, K_{0}=0.158
$$

In the present epoch, we have roughly $5 / 6$ bound matter, and $1 / 6$ source matter. For the universe as a whole, the densities are smeared or average values, valid when distance scales in excess of $100 \mathrm{Mpc}$ are considered. Only then is the cosmos of uniform density, and possesses spherical symmetry. For the entire universe, or for a universe in excess of $100 \mathrm{Mpc}$,

$$
M_{B}=\left(\chi_{0} / K_{0}\right) M_{F}=5.327 M_{F}
$$

We see in Equation (4-12), that the dark matter contribution is 5.327 times the ordinary matter contribution, nothing new, since, $\Omega_{B} / \Omega_{F}=0.2589 / 0.0486=5.327$.

We now focus on Equation (4-6c). With the help of Equations (4-4), and (4-5a, b), this can be rewritten as,

$$
\begin{gathered}
\rho_{g g, 0}=0.6911 \rho_{0} \\
\rho_{A A, 0}\left(1+\chi_{0} / K_{0}\right)=0.6911 \rho_{0} \\
\Omega_{A A, 0} \rho_{0}(1+5.327)=0.6911 \rho_{0}
\end{gathered}
$$

Solving this for, $\Omega_{A A, 0}$, gives

$$
\Omega_{\mathrm{AA}, 0}=0.109
$$

We have made use of Equation (4-8). This $\Omega_{A A, 0}$ value signifies that portion of dark energy, which is due to source matter. The other component of dark energy, $\Omega_{A B, 0}=\Omega_{g g, 0}-\Omega_{A A, 0}=0.6911-0.109=0.582$, is due to polarized, or bound, matter. According to this result, we see that most of the energy content in the present universe is due to gravitational fields set up by, $M_{B}$. That accounts for $58.2 \%$ of the energy content of the universe. The gravitational fields produced by source matter account for another, $10.9 \%$, of the total. The rest is ordinary matter, and dark matter. Only in a relatively cool and very dilute un- 
iverse, can the gravitational field density exceed the ordinary and bound mass density. The, $\rho_{A B, 0}$ is the gravitational field density due to bound mass density, $\rho_{B}$, whereas the $\rho_{A A, 0}$ is the gravitational field density due to source mass density, $\rho_{F}$. Again, all densities are smeared or average values, and should have bars over them.

The gravitational field components in Equations (4-2), and (4-4), are very real, and thus cannot be ignored in the Friedmann equation. By Gauss's law from the last section,

$$
1 / \varepsilon_{0} M_{F}=-\oint \overrightarrow{g^{(0)}} \cdot \overrightarrow{\mathrm{d} A}, 1 / \varepsilon_{0} M_{B}=-\oint \overrightarrow{g^{(1)}} \cdot \overrightarrow{\mathrm{d} A}
$$

If there is a net positive source mass in the cosmos, which we know there is, then obviously, $\overline{g^{(0)}} \neq 0$, by Equation (4-15a). By Equation (4-15b), if there is a net positive bound mass in the cosmos, which is an assumption, then, $\overrightarrow{g^{(1)}} \neq 0$. Equations (4-15a, b), also tell us that,

$$
\overline{g^{(0)}}=G M_{F} / R^{2}=1 / \varepsilon_{0} \sigma_{F}, \overline{g^{(1)}}=G M_{B} / R^{2}=1 / \varepsilon_{0} \sigma_{B}
$$

Spherical symmetry will exist for large distance scales. The, $\sigma_{F}$, and $\sigma_{B}$, are the surface mass densities for both source mass and bound mass, respectively, at the Hubble radius. The total macroscopic gravitational field, $\bar{g}$, is the sum of Equations (4-16a), and (4-16b). The right hand sides of Equations (4-16a), and (4-16b), do not vanish. Therefore, it follows that the left hand sides cannot disappear either.

In the distant past, it is thought that the cosmic susceptibility, $\chi(a)$, equaled zero. Then, the bound mass, $M_{B}$, vanishes, as does, $\overline{g^{(1)}}$. Thus, both, $\rho_{B}$, and $\rho_{A B}$, vanish in this limit. Equations (4-16a, b), indicate surface values for these gravitational fields, and so we cannot use, the above equations to determine how, $\overline{g^{(0)}}$, and $\overline{g^{(1)}}$, scale upon cosmic expansion. Equations (4-16a, b), are formal devices to calculate an average, or smeared, net, $\overline{g^{(0)}}$, and $\overline{g^{(1)}}$ value.

We have seen from Equation (4-14), that, $\Omega_{A A, 0}=0.109$. From Equation (4-5a), it follows that,

$$
\begin{gathered}
\rho_{A A, 0}=0.109 \rho_{0} \\
1 /\left(2 c^{2}\right) \varepsilon_{0}\left(g_{0}^{(0)}\right)^{2}=0.109 \times(8.624 \mathrm{E}-27)
\end{gathered}
$$

In the present epoch, the total $\Lambda \mathrm{CDM}$ mass density equals, $\rho_{0}=8.624 \mathrm{E}-27(\mathrm{MKS})$. Equation (2-17), can be solved for $g_{0}^{(0)}$ since, $\varepsilon_{0}=1 /\left(4 \pi G_{0}\right)=1.192 \mathrm{E} 9(\mathrm{MKS})$. The solution is

$$
g_{0}^{(0)}=\overline{g_{0}^{(0)}}=3.771 \mathrm{E}-10 \mathrm{~m} / \mathrm{s}^{2}
$$

This is the net gravitational field due to all the source masses, $\sum M_{F, i}$, in the universe, when looked at on a grand scale. It is the same gravitational field as in Equation (4-16a). Any observer, no matter where they are located in the universe, would deduce such a value using Gauss's law.

Once we have this gravitational field, $g_{0}^{(0)}$, the remaining gravitational fields 
are easily found. Since, $g_{0}^{(0)}=K_{0} g_{0}=0.158 g_{0}$, we find that

$$
g_{0}=\overline{g_{0}}=2.387 \mathrm{E}-9 \mathrm{~m} / \mathrm{s}^{2}
$$

This is the net macroscopic field due to all masses, free and bound, $\sum M_{F, i}+\sum M_{B, i}$, in the universe. And for the gravitational field due to the bound sources only, we obtain, $g_{0}^{(1)}=\chi_{0} g_{0}=0.842 g_{0}$, or,

$$
g_{0}^{(1)}=\overline{g_{0}^{(1)}}=2.010 \mathrm{E}-9 \mathrm{~m} / \mathrm{s}^{2}
$$

This is due to the sum of all the bound mass, $\sum M_{B, i}=M_{B}$, in the universe. If we were to substitute this mass in Equation (4-16b), we would obtain this gravitational filed.

Let us next calculate the net smeared polarization, $\overline{P_{0}}$, and the net smeared gravitational displacement vector, $\overline{D_{0}}$, in the current epoch. We'll use Equation (3-19a), for the former, and Equation (3-20a), for the latter. We find, respectively,

$$
\begin{gathered}
\overline{P_{0}}=\varepsilon_{0} \chi_{0} \overline{g_{0}}=\varepsilon_{0} \overline{g_{0}^{(1)}}=2.396 \mathrm{~kg} / \mathrm{m}^{2} \\
\overline{D_{0}}=\varepsilon_{0} K_{0} \overline{g_{0}}=\varepsilon_{0} \overline{g_{0}^{(0)}}=0.4495 \mathrm{~kg} / \mathrm{m}^{2}
\end{gathered}
$$

We have substituted the values for the gravitational fields, specified in Equations (4-20), and (4-18), in order to evaluate the numerical results in relations, (4-21a, b). The net polarization and the net gravitational displacement vector, are both average or smeared, quantities holding for the cosmos as a whole. Their sum equals,

$$
\varepsilon_{0} \overline{g_{0}}=\overline{D_{0}}+\overline{P_{0}}=2.846 \mathrm{~kg} / \mathrm{m}^{2}
$$

This can be verified a number of ways, but perhaps the simplest is to add the right hand sides of Equations (4-21a, b), and compare that to the right hand side of Equation (4-19), multiplied by $\varepsilon_{0}$. The results match numerically.

We turn next to the Hubble radius of the observable universe. We can use Equation (3-11), from the last section. Written out, that equation in the present epoch reads,

$$
\overline{g_{0}^{(0)}}=1 /\left(3 \varepsilon_{0}\right) R_{0} \overline{\rho_{F, 0}}
$$

We have specific values for each quantity in this equation except for the Hubble radius, $R_{0}$. Therefore, we can use this equation to solve for, $R_{0}$. For, $\overline{g_{0}^{(0)}}$, we use the numerical result in Equation (4-18). The gravitational permittivity equals, $\varepsilon_{0}=1 /\left(4 \pi G_{0}\right)=1.192 \mathrm{E} 9(\mathrm{MKS})$. And, for, $\overline{\rho_{F, 0}}$, keep in mind that this equals, $\overline{\rho_{F, 0}} \cong \overline{\rho_{b, 0}}=\Omega_{b} \rho_{0}=0.0486 \times(8.624 \mathrm{E}-27)$. We substitute all of this into Equation (4-23), and solve for $R_{0}$. The result is

$$
R_{0}=3.217 \mathrm{E} 27 \text { meters }
$$

This is our estimate for the radius of the observable universe. This is significantly larger than the value often quoted, which is, 4.4E26 meters [30] [31]. In fact, our value is about 7.3 times larger. Our value is obtained as a direct consequence of Gauss's law, and the density parameter values in Friedmann's equa- 
tion. As such, we believe it to be very accurate.

Once the Hubble radius is known, it is possible to determine the various masses with certainty in the universe. For the free mass, or the source mass, as we sometimes refer to it, we find,

$$
M_{F}=4 / 3 \pi R_{0}^{3} \overline{\rho_{F, 0}}=4 / 3 \pi R_{0}^{3} \Omega_{b} \rho_{0}=5.847 \mathrm{E} 55 \mathrm{~kg}
$$

This is about $7.3^{3} \cong 390$ times greater than current estimates, which is about, $1.5 \mathrm{E} 53 \mathrm{~kg}$. This increase in mass is due to our increased radius value. One will note that our values for both Hubble radius, and the ordinary mass in the universe, are very precise, as precise as the determination of density parameter values in Friedmann's equation. We have three decimal point accuracy ( 3 significant figures), versus current estimates (1 significant figure). The bound, or polarized mass, $M_{B}$, is 5.327 times greater than the free mass, which is specified by Equation (4-25). See Equation (4-12).

We close this section with an observation relating to the Friedmann equation. One may ask the question, where is the planckion mass density in Friedmann's equation? A quick answer would be that there is none, because the positive mass planckions neutralize, and balance out, the negative mass planckions. But this is only true for an undisturbed vacuum. The vacuum we see in the cosmos today is not totally neutral. It is disturbed because there is net positive macroscopic mass in the universe, as well as bound mass relating to the former. Bound mass is an assumption, in this paper, but source mass is a given. The planckion mass density is, actually, the dark energy mass density, $\rho_{g g}$. And, since, $\rho_{g g} \neq 0$, there is actually a net planckion mass density. To see this we argue as follows.

Consider a universe where we have $N_{+}$positive mass planckions, and $N_{-}$ negative mass planckions. The mass density associated with each species adds up to,

$$
\begin{aligned}
\rho_{P l} & =N_{+} M_{P l} / \text { Volume }+N_{-}\left(-M_{P l}\right) / \text { Volume } \\
& =\left(n_{+}-n_{-}\right) M_{P l}
\end{aligned}
$$

This is nothing else but Equation (2-4). However, the right hand side only equals zero for an undisturbed vacuum.

If there is a net gravitational field in the universe, which we know there is, because there is net source mass, and possibly bound mass, as well, then how would that net gravitational field manifest itself? The answer is through a mass density, $\rho_{g g}$. And, the $\rho_{g g}$ value, quite literally, represents mass creation, specifically within the vacuum, where there originally was none. Since the vacuum is thought to be made up of planckions, it can only be planckions which create that "extra" mass per unit volume. We are therefore justified in setting,

$$
\rho_{P l}=\rho_{g g}
$$

This unusual equation will be put to work in later papers. Again, it can only be planckions which are responsible for any mass density creation within the vacuum, if they and blackbody photons make up the vacuum, and Equation (4-27), expresses this fact. Since, $\rho_{g g}$, equals, $\rho_{\Lambda}$, we can also claim that, $\rho_{P l}=\rho_{\Lambda}$. 
We believe that Planckion mass density may very well equal dark energy density.

This brings us to an interesting observation. The right hand side of Equation (4-26), does not vanish. Moreover,

$$
\left(n_{+}-n_{-}\right) M_{P l}=\rho_{g g}=\rho_{\Lambda}=0.6911 \times(8.624 \mathrm{E}-27)
$$

The current value of, $M_{P l}$, equals, $M_{P l}=2.176 \mathrm{E}-8 \mathrm{~kg}$. We substitute this into Equation (4-28), and rework it to find that,

$$
\left(n_{+}-n_{-}\right)=2.74 \mathrm{E}-19 \text { meters }^{-3}
$$

There appears to be a slight imbalance between positive and negative planckion number density within the cosmos! The number densities in Equation (4-29), are average, or smeared values, holding for the universe as a whole. The reason for the imbalance in planckion number density in the current epoch, is unknown. Possibly, it may have to do with elementary particle formation and ordinary mass creation within the universe. When positive and negative planckions first condensed out of the vacuum, it is reasonable to assume that their respective total numbers matched, leading to a perfect balance in number density. This is a topic for future work.

\section{Summary and Conclusions}

We presented a model of gravitational polarization based on previous work of Hajdukovic, and Winterberg. We borrowed essential ideas from each author, and combined them in such a way so as to create a new theory for dark matter, and dark energy. In our theory, dark matter and dark energy are related. Their common origin is due to a two-component superfluid/supersolid, made up of submicroscopic positive and negative mass particles, called planckions. These planckions fill all of space and interact according to specific fluid forces within their species. The masses involved have the value, $\pm M_{P l}=2.716 \mathrm{E}-8$ (current era), which we recognize as positive and negative the Planck mass. Like planckions, whether they have positive or negative mass, simultaneously repel and attract one another, maintaining a fixed distance of separation between them. Unlike planckions do not interact directly, but indirectly, as they are forced next to one other due to their species specific fluid forces.

This vast assembly (sea) of positive and negative mass particles leads to a mass compensating effect, giving space the illusion that space is seemingly empty, when, in fact, it is not. The reasons for formulating such a space have been presented, and worked out in great detail, by Winterberg. We focused in this paper of trying to apply his ideas to expansion, and specifically, the density parameters, within the Friedmann equation. In the $\Lambda$ CDM model, no specific interconnection seems to exist between dark matter, dark energy, and ordinary matter. We provide such a connection, inter-relating all three ideas. This is an extension of Winterberg's model, leading to new results, and insights.

A space made up of positive and negative mass planckions can be gravitationally polarized if conditions are right. And it is precisely the polarization of space, 
and bound mass formation, which leads to dark matter. There are also gravitational fields, because matter fills the universe. According to Gauss's law, we have a net surface gravitational field, $\overline{g_{0}^{(0)}}$, which is due to ordinary source mass, a given. We also have a net surface gravitational field, $\overline{g_{0}^{(1)}}$, associated with bound, or polarized, matter, an assumption. And because any observer within the observable universe will measure the same values for each, irrespective of location, they must hold point for point within the cosmos. The gravitational fields, $\overline{g_{0}^{(0)}}$, and $\overline{g_{0}^{(1)}}$, are smeared or average values, holding for the cosmos as a whole, and these components add up to, $\overline{g_{0}}$, which is the macroscopic gravitational field. We assume uniform density for matter for distance scales beyond, $100 \mathrm{Mpc}$. Dark energy is identified by us as the mass density associated with these gravitational fields, which permeate all of space. Specifically, we set, $\rho_{\Lambda}=\rho_{g g}=1 /\left(2 c^{2}\right) K \varepsilon \bar{g}^{2}$, by analogy to electrostatics. In this equation, $\bar{g}$, is the smeared gravitational field, which includes contributions from both source and bound mass, $K$ equals the relative permittivity, and $\varepsilon$ is the gravitational permittivity, defined as, $\varepsilon \equiv 1 /(4 \pi G)$. See Equations (4-3), (4-4), and (4-5).

The assumed gravitational polarization, in our model, is due to the positive and negative mass planckions making up the vacuum. When cosmic CBR temperatures are low enough, these material particles can form gravitational dipoles, which can be ordered/aligned spatially to create a net polarization of the surrounding space. This polarization cloud formed around ordinary matter leads to a mass density, $\rho_{B}=-\vec{\nabla} \cdot \vec{P}$, which we identified as dark matter. The polarization leads to increased mass, and can explain many of the characteristics usually associated with dark matter, such as the halo effect around galaxies, rotation curves within galaxies, virial motion of galaxies within superclusters, gravitational lensing, etc.

We started in Section II by looking at the fluid forces acting on the individual planckions, and showed how this led to a spatial anchoring of planckions. Restoring forces were assumed, which causes individual planckions to maintain a fixed distance of separation from one another. Those restoring forces were presented in Equations (2-9), and (2-10), which are new. The positive (negative) planckion number density will increase (decrease) with increasing, $x$, the displacement from equilibrium. The $\kappa$ refers to the positive and negative mass planckion spring constant, and, $n_{+}(0)=n_{-}(0)$, are the number densities for an undisturbed vacuum. Planckions oscillate and vibrate with a frequency given by Equation (2-12), and the spring constant scales according to Equation (2-16), upon the expansion of the universe. The, $\kappa$, has a very large value in the present epoch indicating a very stiff medium, i.e., a very stiff vacuum. Spatial anchoring is needed for dipole formation, and polarization, among other reasons.

In section III, we presented a whole new theory of gravistatics by analogy to electrostatics. Anti-screening in gravistatics is a new feature. A table, summarizing some of the important similarities, and differences, between electrostatics 
and gravistatics, is given in Table 1. Many of the concepts can be carried over directly from electrostatics to gravistatics, including the definition of a "gravitic", the gravitational equivalent of a dielectric. We also have gravitational bound mass, gravitational susceptibility, and gravitational polarization. The gravitic is our vacuum, populated with positive and negative mass planckions.

In Section IV, specific identifications for dark matter and dark energy were made. Dark matter was identified with bound, or polarized, mass, set up within the vacuum or gravitic. This is matter trapped in dipole formation and macroscopic ordering/alignment is necessary. The dipoles have to organize themselves in some fashion in order to create a net macroscopic effect. For the specific identifications, see Equation (4-6b), which holds for dark matter. Ordinary, or source matter, made up of quarks and leptons, is specified in Equation (4-6a). And, for dark energy, refer to Equation (4-6c). When written out, dark energy can be expressed as in Equation (4-3), or, as in Equations (4-4), with (4-5). Numerically, using the $\Lambda \mathrm{CDM}$ density parameter values in Friedmannn's equation, we found that in the current epoch, the smeared susceptibility of the cosmos is, $\overline{\chi_{0}}=0.842$. See Equation (4-11a). The relative permittivity equals, $\overline{K_{0}}=0.158$, which is Equation (4-11b). We also calculated the net gravitational fields permeating the vacuum. These are given by Equations (4-18), (4-19), and (4-20). The net polarization of the space, when taken as a whole equals, $\overline{P_{0}}=\varepsilon_{0} \chi_{0} \overline{g_{0}}=\varepsilon_{0} \overline{g_{0}^{(1)}}=2.396 \mathrm{~kg} / \mathrm{m}^{2}$, which is Equation (4-21a).

Our gravitational model leads to new values for the Hubble radius, and for baryonic mass in the observable universe. We obtain Equations (4-24), and (4-25), respectively. These new values are highly precise because they are direct consequences of the density parameter values in Friedmann's equation, and Gauss's law. We have three significant figures for each quantity. Finally, we theorized that the planckion mass density is nothing else but the dark energy mass density. See Equations (4-27), (4-28), and (4-29). In the current epoch, this leads to a net imbalance in the number density for planckions. The positive mass planckions are greater in number than the negative mass planckions. The reasons for this are unknown, but probably have something to do with elementary particle formation, and net mass aggregation in the cosmos.

\section{Acknowledgements}

The author would like to thank his parents, Agnes and Herbert Pilot, his first, and, most effective, teachers. Without them, I could have never fulfilled my dreams.

\section{Conflicts of Interest}

The author declares no conflicts of interest regarding the publication of this paper.

\section{References}

[1] Maeder, A., et al. (Planck Collaboration) (2018) Planck 2018 Results. VI. Cosmo- 
logical Parameters.

[2] Tanabashi, M., et al. (Particle Data Group) (2019) Astrophysical Constants and Parameters. Physical Review D, 98, Article ID: 030001.

https://doi.org/10.1103/PhysRevD.98.030001

[3] Planck Collaboration (2016) Planck 2015 Results. XIII. Cosmological Parameters. Astronomy \& Astrophysics, 594, A13. https://doi.org/10.1051/0004-6361/201525830

[4] Hajdukovic, D. (2011) Is Dark Matter an Illusion Created by the Gravitational Polarization of the Quantum Vacuum? Astrophysics and Space Science, 334, 215-218. https://link.springer.com/article/10.1007/s10509-011-0744-4 https://arxiv.org/ftp/arxiv/papers/1106/1106.0847.pdf https://doi.org/10.1007/s10509-011-0744-4

[5] Hajdukovic, D. (2015) What If Quantum Vacuum Fluctuations Are Virtual Gravitational Dipoles? The 3rd International Workshop on Antimatter and Gravity, London, 4-7 August 2015, 1-9.

https://indico.cern.ch/event/361413/contributions/1776293/attachments/1135100/1 623933/WAG 2015 UCL Hajduk.pdf

[6] Hajdukovic, D. (2016) Quantum Vacuum as the Cause of the Phenomena Usually Attributed to Dark Matter.

https://indico.cern.ch/event/336103/contributions/786848/attachments/1204251/17 98916/DM-Hajdukovic.proc.pdf

[7] Hajdukovic, D. (2020) On the Gravitational Field of a Point-Like Body Immersed in Quantum Vacuum. Monthly Notices of the Royal Astronomical Society, 491, 4816-4828. https://hal.archives-ouvertes.fr/hal-02087886/document

[8] Winterberg, F. (2003) Planck Mass Plasma Vacuum Conjecture. Zeitschrift für Naturforschung $A, 58,231$. https://doi.org/10.1515/zna-2003-0410

[9] Winterberg, F. (2002) Planck Mass Rotons as Cold Dark Matter and Quintessence. Zeitschrift für Naturforschung A, 57, 202-204.

https://doi.org/10.1515/zna-2002-3-414

[10] Winterberg, F. (1995) Derivation of Quantum Mechanics from the Boltzmann Equation for the Planck Aether. International Journal of Theoretical Physics, 34, 2145. https://doi.org/10.1007/BF00673076

[11] Winterberg, F. (1998) The Planck Aether Model for a Unified Theory of Elementary Particles. International Journal of Theoretical Physics, 33, 1275.

https://doi.org/10.1007/BF00670794

[12] Winterberg, F. (1993) Physical Continuum and the Problem of a Finitistic Quantum Field Theory. International Journal of Theoretical Physics, 32, 261. https://doi.org/10.1007/BF00673716

[13] Winterberg, F. (1992) Cosmological Implications of the Planck Aether Model for a Unified Field Theory. Zeitschrift für Naturforschung Physical Sciences, 47, 1217. https://doi.org/10.1515/zna-1992-1207

[14] Winterberg, F. (2002) The Planck Aether Hypothesis. Monograph, The C.F. Gauss Academy of Science Press, Reno.

[15] Weinberg, S. (1989) The Cosmological Constant Problem. Reviews of Modern Physics, 61, 1. https://doi.org/10.1103/RevModPhys.61.1

[16] Würsten, F. (2017) Crystalline and Liquid at the Same Time. ETH Zurich. https://ethz.ch/content/main/en/news-und-veranstaltungen/eth-news/news/2017/03 $\underline{\text { Kristallin-und-fluessig-zugleich.html }}$ 
[17] Léonard, J., Morales, A., Zupancic, P., Esslinger, T. and Donner, T. (2017) Supersolid Formation in a Quantum Gas Breaking a Continuous Translational Symmetry. Nature, 543, 87-90. https://doi.org/10.1038/nature21067

[18] Keller, J.C. (2017) MIT Researchers Create New Form of Matter. MIT News. https://news.mit.edu/2017/mit-researchers-create-new-form-matter-0302

[19] Li, J.-R., Lee, J., Huang, W.J., Burchesky, S., Shteynas, B., Top, F.Ç., Jamison, A.O. and Ketterle, W. (2017) A Stripe Phase with Supersolid Properties in Spin-Orbit-Coupled Bose-Einstein Condensates. Nature, 543, 91-94. https://doi.org/10.1038/nature21431

[20] Donner, T. (2019) Viewpoint: Dipolar Quantum Gases Go Supersolid. Physics, 12, 38. https://physics.aps.org/articles/v12/38 https://doi.org/10.1103/Physics.12.38

[21] Guo, M.Y., Böttcher, F.B., Hertkorn, J., Schmidt, J.-N., Wenzel, Matthias, B., Hans, P., Langen, T. and Pfau, T. (2019) The Low-Energy Goldstone Mode in a Trapped Dipolar Supersolid. Nature, 574, 386-389.

https://doi.org/10.1038/s41586-019-1569-5

[22] Tanzi, L., Roccuzzo, S.M., Lucioni, E., Famà, F., Fioretti, A., Gabbanini, C., Modugno, G., Recati, A. and Stringari, S. (2019) Supersolid Symmetry Breaking from Compressional Oscillations in a Dipolar Quantum Gas. Nature, 574, 382-385. https://doi.org/10.1038/s41586-019-1568-6

[23] Corda, C. (2009) Interferometric Detection of Gravitational Waves: The Definitive Test for General Relativity. International Journal of Modern Physics D, 18, 2275-2282. https://doi.org/10.1142/S0218271809015904

[24] Pilot, C. (2018) Is Quintessence an Indication of a Time-Varying Gravitational Constant? Journal of High Energy Physics, Gravitation and Cosmology, 5, 41-81. https://www.scirp.org/journal/paperinformation.aspx?paperid=88999

[25] Pilot, C. (2019) The Age of the Universe Predicted by a Time Varying G? Journal of High Energy Physics, Gravitation and Cosmology, 5, 928-934. https://www.scirp.org/Journal/paperinformation.aspx?paperid=94065 https://doi.org/10.4236/jhepgc.2019.53048

[26] Pilot, C. (2020) Inflation and Rapid Expansion in a Variable G Model. International Journal of Astronomy and Astrophysics, 10, 334-345. https://www.researchgate.net/publication/342988030 Inflation and Rapid Expansi on in a Variable G Model slightly revised version https://doi.org/10.4236/ijaa.2020.104018

[27] Bertotti, B., Balbinot, R., Bergia, S. and Messina, A. (1990) Modern Cosmology in Retrospect. Cambridge University Press, Cambridge, 145.

[28] Matzner, R.A. (1968) On the Present Temperature of Primordial Blackbody Gravitational Radiation. Astrophysical Journal, 154, 1123. https://doi.org/10.1086/149831

[29] Weinberg, S. (1972) Gravitation and Cosmology: Principles and Applications of the General Theory of Relativity. Wiley-VCH, Hoboken.

[30] Bars, I. and Terning, J. (2009) Extra Dimensions in Space and Time. Springer, Berlin. https://doi.org/10.1007/978-0-387-77638-5

[31] Lineweaver, C. and Davis, T.M. (2005) Misconceptions of the Big Bang. Scientific American, 292, 24. 